\title{
Internações por condições sensíveis à atenção primária em Novo Hamburgo, Rio Grande do Sul
}

\author{
Hospitalizations for primary care sensitive conditions in Novo Hamburgo, Rio Grande do Sul \\ Hospitalizaciones por condiciones sensibles a la atención primaria en Novo Hamburgo, \\ Rio Grande do Sul
}

Jocinei Santos de Arruda ${ }^{a}$, Juvenal Soares Dias Da Costa ${ }^{a}$

\section{Resumo}

Objetivos: O presente estudo teve por objetivo analisar a tendência das internações por condições sensíveis à atenção primária (ICSAP) em Novo Hamburgo, comparando-a com o restante do Estado do Rio Grande do Sul, e relacionando-a com os investimentos financeiros em saúde e a cobertura da Estratégia Saúde da Família (ESF) no município, no período de 1998 a 2012. Métodos: Foi realizado um estudo ecológico. Os dados foram coletados do Sistema de Internações Hospitalares do SUS. As causas de ICSAP foram baseadas na lista do Ministério da Saúde. A tendência das ICSAP em Novo Hamburgo e no restante do Estado do Rio Grande do Sul foram analisadas por meio dos seus coeficientes padronizados, pelas médias móveis a cada três anos e pela Regressão Binomial Negativa. Foi realizada análise de correlação entre as variáveis de interesse. Resultados: Apesar do aumento de $329,26 \%$ nos investimentos financeiros e da expansão da cobertura por ESF no período, atingindo $20,20 \%$ da população, não houve diminuição nas tendências dos coeficientes de ICSAP em Novo Hamburgo. No restante do Rio Grande do Sul houve redução nas ICSAP, contudo, os coeficientes foram maiores do que em Novo Hamburgo no período estudado. Conclusões: Embora no presente estudo não tenha sido encontrada associação entre a diminuição nos coeficientes de ICSAP com o aumento da cobertura pela ESF, incremento do investimento financeiro em saúde, investimento financeiro em APS e o gasto per capita em saúde, há evidências que estes estejam correlacionados. Provavelmente, a cobertura de ESF não foi suficiente para impactar as ICSAP.

\section{Abstract}

Objectives: This study aimed to analyze trends in admissions for ambulatory care sensitive conditions (ACSC) in Novo Hamburgo, comparing it with the rest of the Rio Grande do Sul State, and relating it to the investments in health and the Family Health Strategy (FHS) coverage in the city, from 1998 to 2012. Methods: We conducted an ecological study. Data were obtained from the Unified Health System's Hospital Database (SIH-SUS). The causes of ACSC were based on a national list published by the Ministry of Health. ACSC tendencies in Novo Hamburgo and the rest of the Rio Grande do Sul State were analyzed by means of their standardized rates, based on moving averages every three years, and Negative Binomial Regression. Correlation analysis was performed between the variables of interest. Results: Despite an increase of $329.26 \%$ in financial investments and the expansion of FHS coverage in the period, reaching $20.20 \%$ of the population, there was no decrease in ACSC rate trends in Novo Hamburgo. In the rest of Rio Grande do Sul, there was a reduction in ACSC, however, the rates were higher than in Novo Hamburgo during the same period. Conclusion: Although this study found no association between the decrease in ACSC rates and increased coverage for FHS, increase financial investment in health, financial investment in primary care and per capita spending on health, there is evidence that they are correlated. Probably the FHS coverage was not enough to impact the ACSC.

Como citar: Arruda JS, Dias da Costa JS. Internações por condições sensíveis à atenção primária em Novo Hamburgo, Rio Grande do Sul. Rev Bras Med Fam Comunidade. 2017;12(39):1-11. http://dx.doi.org/10.5712/rbmfc12(39)1256
Palavras-chave:

Qualidade da

Assistência à Saúde

Atenção Primária à Saúde

Hospitalização

Saúde da Família

Financiamento da

Assistência à Saúde

Keywords:

Quality of Health Care

Primary Health Care

Hospitalization

Family Health

Health Care Financing
Fonte de financiamento: declaram não haver. Parecer CEP: 127/2014 (UNISINOS), aprovado em 11/09/2014. Conflito de interesses: declaram não haver. Procedência e revisão por pares: revisado por pares. Recebido em: 22/12/2015. Aprovado em: 23/04/2017. 


\section{Resumen}

Objetivos: Este estudio tuvo como objetivo analizar las tendencias de las hospitalizaciones por condiciones sensibles a cuidados de atención primaria (HCSAP) en Novo Hamburgo, comparándolo con el resto del estado de Río Grande do Sul, y relacionándolo con las inversiones en la salud y la cobertura de la Estrategia Salud de la Familia (ESF) en la ciudad, de 1998 a 2012. Métodos: Se realizó un estudio ecológico. Los datos se obtuvieron a través del sistema SUS de los ingresos hospitalarios. Las causas de HCSAP se basaron en la lista del Ministerio de la Salud. La tendencia de HCSAP en Novo Hamburgo y el resto del Rio Grande do Sul se analizaron por medio de los coeficientes estandarizados, las medias móviles cada tres años y la Regresión Binomial Negativa. Se realizó análisis de correlación entre las variables de interés. Resultados: A pesar de un aumento de un 329,26\% en las inversiones financieras y la ampliación de la cobertura de ESF en el período, llegando a un $20,20 \%$ de la población, no hubo una disminución en las tendencias de las tasas de HCSAP. En el resto de Rio Grande do Sul hubo una reducción en HCSAP, sin embargo, las tasas fueron más altas que en Novo Hamburgo, en el mismo período. Conclusión: Aunque en este estudio no se encontró asociación entre la disminución de los coeficientes HCSAP con el aumento de la cobertura por el ESF, con el aumento de la inversión financiera en APS, y con el gasto per cápita en salud, hay evidencia de que están correlacionados. Es probable que la cobertura del ESF no fue suficiente para afectar la HCSAP.
Palabras clave:

Calidad de la Atención

en Salud

Atención Primaria de Salud

Hospitalización

Salud de la Familia

Financiación de la

Atención de la Salud

\section{Introdução}

Na década de 1990, pesquisadores de países como Estados Unidos,,$^{1,2}$ Espanha $^{3}$ e Canadá passaram $^{2}$ a realizar estudos utilizando um novo indicador, denominado internações por condições sensíveis à atenção primária (ICSAP). Esse indicador definiu um grupo de doenças para as quais um cuidado efetivo no nível da atenção primária diminuiria o risco das hospitalizações, tanto na prevenção do surgimento dos agravos quanto no manejo adequado das condições ou patologias crônicas. ${ }^{5}$ Desta forma, o indicador serviria para avaliação da atenção primária, bem como de todo sistema de saúde e poderia prever gastos, necessidades de investimento e definir prioridades em saúde.

Em 2008, o Ministério da Saúde do Brasil elaborou uma lista própria de condições sensíveis à atenção primária, como tentativa de avaliar a qualidade do nível de atenção básica. ${ }^{6}$ Com a elaboração dessa lista, tem sido possível disseminar estudos avaliativos sobre os sistemas de atenção primária em diferentes regiões do país, ${ }^{7-10}$ a partir do princípio de que elevadas taxas de ICSAP indicariam problemas de acesso ou de efetividade dos cuidados em atenção primária à saúde (APS). ${ }^{5,11}$

Com o objetivo da mudança no modelo de atenção até então vigente no Brasil, em 1994 foi lançado o Programa Saúde da Família, atualmente transformado em Estratégia Saúde da Família (ESF). Essa estratégia surgiu como tentativa de reorganizar a utilização dos níveis assistenciais de forma a racionalizar o atendimento, contribuindo para a melhoria do acesso e da qualidade da APS. ${ }^{6}$

Contudo, sabe-se que os investimentos financeiros realizados em saúde no Brasil, nos três níveis de atenção, ainda são insuficientes. Destaca-se que há uma desigualdade em investimentos, tendo em vista a maior escassez de recursos principalmente na atenção primária, devido a uma visão distorcida da sua complexidade, que leva à desvalorização deste nível de atenção. ${ }^{12-14}$

As ICSAP, como indicador de acesso e qualidade da atenção, passou a valorizar a APS, servindo de ferramenta para avaliação do desempenho e seu reflexo nos investimentos em saúde. ${ }^{6}$ Alguns estudos já foram realizados nesse sentido; no interior de São Paulo, entre 2008 e 2010, as ICSAP geraram um gasto de $R \$ 30.370 .691,08$, o que representou $17 \%$ dos investimentos financeiros totais realizados em saúde. ${ }^{15}$ Em outro estudo relacionado aos gastos com ICSAP, foi demonstrada uma redução no percentual dos gastos no estado de São Paulo, entre 2000 e 2007, de 14,7\%, em 2000, para 13,8\%, em $2007 .{ }^{16}$ 
O presente estudo teve por objetivo analisar a tendência das ICSAP em Novo Hamburgo, comparando-a com o restante do Estado do Rio Grande do Sul, e relacionando-a com os investimentos financeiros em saúde e a cobertura da ESF no município, no período de 1998 a 2012.

\section{Métodos}

Esse trabalho trata-se de um estudo ecológico com o objetivo de analisar a tendência por ICSAP no município de Novo Hamburgo, comparando-a com o restante do Estado do Rio Grande do Sul, no período de 1998 a 2012.

De acordo com o Censo no ano de 2010, o município de Novo Hamburgo possuía 238.940 habitantes. Nesse município, as principais portas de acesso de atenção primária à saúde são 14 Unidades de Saúde da Família (USF) e 2 Unidades Básicas de Saúde (UBS). Além disso, também são oferecidos atendimentos especializados em dois centros de saúde. Os atendimentos de urgência e emergência são ofertados em duas unidades mantidas pelo município, uma destas com estrutura de Unidade de Pronto Atendimento (UPA). Além disso, em Novo Hamburgo, os atendimentos contam com o suporte de um hospital municipal (266 leitos) e um hospital conveniado (53 leitos).

Os dados secundários foram coletados do DATASUS (www.datasus.gov.br), por meio do Sistema de Informações Hospitalares do Sistema Único de Saúde (SIH/SUS), e as informações populacionais foram disponibilizadas pelo Instituto Brasileiro de Geografia e Estatística (IBGE).

As causas de ICSAP foram baseadas na lista nacional, conforme a Portaria do Ministério da Saúde no 221 , de 17 de abril de 2008.

Assim, foram construídos os coeficientes brutos de ICSAP. Para comparação das tendências, também foram elaborados os coeficientes brutos por todas as causas de internação, excluídas as causas obstétricas. Posteriormente, foi realizada a padronização direta dos coeficientes para retirar a influência das diferenças devido às estruturas etárias, utilizando-se como referência a população do Estado do Rio Grande do Sul no ano de $2010 .{ }^{17}$ Com a finalidade de atenuar as variações aleatórias, utilizou-se a técnica denominada média móvel a cada três anos. ${ }^{18}$

Assim, a tendência das ICSAP em Novo Hamburgo e no restante do Estado do Rio Grande do Sul foram analisadas por meio dos seus coeficientes padronizados, ${ }^{17}$ bem como das médias móveis a cada três anos. ${ }^{18}$ As tendências dos coeficientes padronizados e das médias móveis foram inicialmente analisadas pelas diferenças percentuais.

Os dados financeiros de Novo Hamburgo foram extraídos dos extratos de repasses financeiros disponíveis no Sistema de Informações sobre Orçamentos Públicos em Saúde (www.saude.gov.br/siops). Os indicadores foram elaborados a partir da despesa liquidada em cada ano da administração direta em saúde. Assim, foi determinado o investimento financeiro/ano, o gasto per capita em saúde, o investimento em APS/ano; esse último disponível desde 2002. O custo anual das ICSAP foi obtido por meio do DATASUS, com isso, descreveu-se o seu percentual em relação ao investimento financeiro/ano.

Para correções em decorrência da inflação no período, no investimento financeiro em saúde/ano, no investimento em atenção primária à saúde/ano e no custo das ICSAP/ano foi utilizado o Índice Geral de Preços: Disponibilidade Interna (IGP-DI), da Fundação Getúlio Vargas. ${ }^{19}$ Já para a padronização e correção 
do gasto per capita em saúde, foi utilizada como moeda padrão o dólar americano, usando na conversão a cotação da moeda na metade do período. ${ }^{20}$

O percentual de cobertura populacional pela ESF anual, em Novo Hamburgo, foi obtido pelas informações fornecidas pelo Departamento de Atenção Básica do Ministério da Saúde (www.dab.saude. gov.br) e, posteriormente, os dados foram tabulados no programa $\mathrm{Exce}^{\circledR}$.

Além disso, por meio da Regressão Binomial Negativa, utilizando o Programa Stata ${ }^{\circledR}$ 11.0, foram analisados os coeficientes padronizados das ICSAP, investimento financeiro, investimento em APS, gasto per capita e a cobertura da ESF no município de Novo Hamburgo. Os resultados foram descritos mediante o coeficiente da razão das médias com seus respectivos intervalos de confiança a 95\% e os resultados dos testes estatísticos. ${ }^{21}$

Realizou-se também a correlação de Spearman, atendendo seus pressupostos, para testar a associação das ICSAP com os investimentos financeiros, investimentos em APS, gastos per capita em saúde e a cobertura da ESF no município de Novo Hamburgo, sendo considerada como significativa quando alcançou valor superior a 0,50 e valor $p$ menor que $0,05 .{ }^{22}$

O projeto foi aprovado pelo Comitê de Ética em Pesquisa da Universidade do Vale do Rio dos Sinos (UNISINOS), mediante resolução 127/2014 de 11/09/2014.

\section{Resultados}

Por meio da análise das ICSAP no município de Novo Hamburgo, constatou-se que o menor coeficiente padronizado foi no ano de 2002 e o maior no ano de 2006, sendo estas, respectivamente, 9,41/1000 e 13,11/1000. Essa variação representou um aumento de $39,31 \%$ das ICSAP. Em relação à análise das ICSAP no Rio Grande do Sul, excluindo Novo Hamburgo, foi demonstrado o maior coeficiente padronizado no ano de 1998 (29,40/1000) e o menor em 2012 (15,95/1000). A variação nos coeficientes padronizados de ICSAP representou redução de 45,75\%. Apesar do aumento encontrado os coeficientes de ICSAP em Novo Hamburgo, esses foram mais baixos do que o restante do Estado em todo o período (Tabela 1).

Quanto à análise dos coeficientes padronizados das internações por todas as causas em Novo Hamburgo, o menor valor encontrado foi no ano de 1998, com 41,73 internações por 1000 habitantes, enquanto que o maior foi em 2012 (55,23/1000), correspondendo a um aumento de 32,35\%. Em relação à análise dos coeficientes padronizados das internações por todas as causas no Rio Grande do Sul, excluindo Novo Hamburgo, constatou-se que o maior valor encontrado foi em 1999 (73,49/1000), enquanto que o menor foi em 2011, com 55,60 internações por 1000 habitantes, correspondendo a uma redução de $24,34 \%$. Apesar do aumento dos coeficientes por internações por todas as causas em Novo Hamburgo, esses foram menores que os verificados no restante do Estado em todo o período (Tabela 1).

Posteriormente, realizou-se a análise das médias móveis de três anos, das internações por todas as causas em Novo Hamburgo, na qual foi possível observar também uma elevação. O menor coeficiente foi observado no ano de 2000 (45,19/1000) e o maior em 2012 (52,39/1000), correspondendo a uma elevação de 15,93\%. Em relação à análise das médias móveis das internações por todas as causas no restante do Rio Grande do Sul, foi possível observar redução nas variações aleatórias. A maior taxa foi observada em 2000 (71,63/1000) e a menor em 2012 (57,03/1000), correspondendo a uma redução de 20,38\% (Gráfico 1). 
Tabela 1. Coeficiente de internações por causas sensíveis em atenção primária (ICSAP) por ano, coeficientes de internações totais por ano ajustados do município de Novo Hamburgo e do estado do Rio Grande do Sul (sem Novo Hamburgo), 1998 a 2012.

\begin{tabular}{lcccc}
\hline Ano & \multicolumn{2}{c}{ Novo Hamburgo } & \multicolumn{2}{c}{ RS (sem Novo Hamburgo) } \\
\cline { 2 - 5 } & $\begin{array}{c}\text { Coeficiente } \\
\text { Ajustado } \\
\text { ICSAP }\end{array}$ & $\begin{array}{c}\text { Coeficiente } \\
\text { Ajustado } \\
\text { Intern. Total }\end{array}$ & $\begin{array}{c}\text { Coeficiente } \\
\text { Ajustado } \\
\text { ICSAP }\end{array}$ & $\begin{array}{c}\text { Coeficiente } \\
\text { Ajustado } \\
\text { Intern. Total }\end{array}$ \\
\hline 1998 & 11,66 & 41,73 & 29,40 & 72,30 \\
1999 & 12,49 & 44,92 & 28,67 & 73,49 \\
2000 & 11,80 & 48,92 & 25,20 & 69,11 \\
2001 & 10,32 & 47,47 & 24,52 & 68,11 \\
2002 & 9,41 & 43,11 & 22,70 & 67,54 \\
2003 & 10,69 & 45,06 & 20,74 & 65,45 \\
2004 & 12,96 & 51,18 & 20,25 & 65,06 \\
2005 & 12,69 & 49,23 & 19,80 & 62,92 \\
2006 & 13,11 & 48,45 & 19,88 & 64,06 \\
2007 & 11,91 & 46,84 & 17,51 & 58,34 \\
2008 & 9,89 & 42,98 & 17,26 & 57,62 \\
2009 & 11,02 & 46,94 & 17,62 & 58,50 \\
2010 & 11,72 & 50,63 & 17,47 & 58,32 \\
2011 & 11,78 & 51,32 & 16,30 & 55,60 \\
2012 & 12,68 & 55,23 & 15,95 & 57,18 \\
\hline
\end{tabular}

Em relação às médias móveis das ICSAP, em Novo Hamburgo, observou-se uma elevação, com o menor coeficiente no ano de 2003 (10,14/1000) e o maior em 2006 (12,92/1000), o que representou um aumento de $27,41 \%$. A análise das médias móveis no restante do Rio Grande do Sul demonstrou uma diminuição nas variáveis aleatórias, com a maior taxa em 2000 (27,75/1000) e a menor no ano de 2012 (16,57/1000), o que representou uma redução de 40,28\% (Gráfico 1).

$\mathrm{Na}$ análise da tendência observou-se que tanto as internações por todas as causas como as ICSAP tiveram decréscimo gradativo ao longo de todo período estudado, 1998 a 2012, no restante do Rio Grande do Sul. Já em relação às tendências no município de Novo Hamburgo, observou-se uma gradativa elevação das internações por todas as causas e uma tendência à estabilização das ICSAP no decorrer do referido período. Ainda assim, os coeficientes padronizados das ICSAP em Novo Hamburgo sempre estiveram abaixo do restante do Estado (Gráfico 1).

Quanto aos investimentos financeiros realizados em saúde, foi possível observar um incremento nos valores aplicados. No ano de 1998 foram investidos $R \$ 30.500 .995,07$ e no ano de 2012 os valores atingiram $\mathrm{R} \$ 130.929 .810,25$, representando um aumento de $329,26 \%$. Observou-se um comportamento semelhante nos gastos per capita em saúde, que tiveram um importante aumento, passando de U\$32,66 em 1998 para U\$275,02 em 2012, correspondendo a um aumento de 742,07\% (Tabela 2).

Os dados referentes aos investimentos financeiros em APS só estavam disponíveis a partir do ano de 2002, quando foram investidos $R \$ 23.215 .162,48$, enquanto que, em 2012, foram investidos $R \$$ 119.990.755,51 em APS. Esta variação correspondeu a um aumento de 416,86\%. Assim, ao relacionar os investimentos em APS com os investimentos totais em saúde, a proporção destinada à APS passou de $50,78 \%$ em 2002 para $91,64 \%$ em 2012, correspondendo a um aumento de $80,46 \%$ no período (Tabela 2 ). 

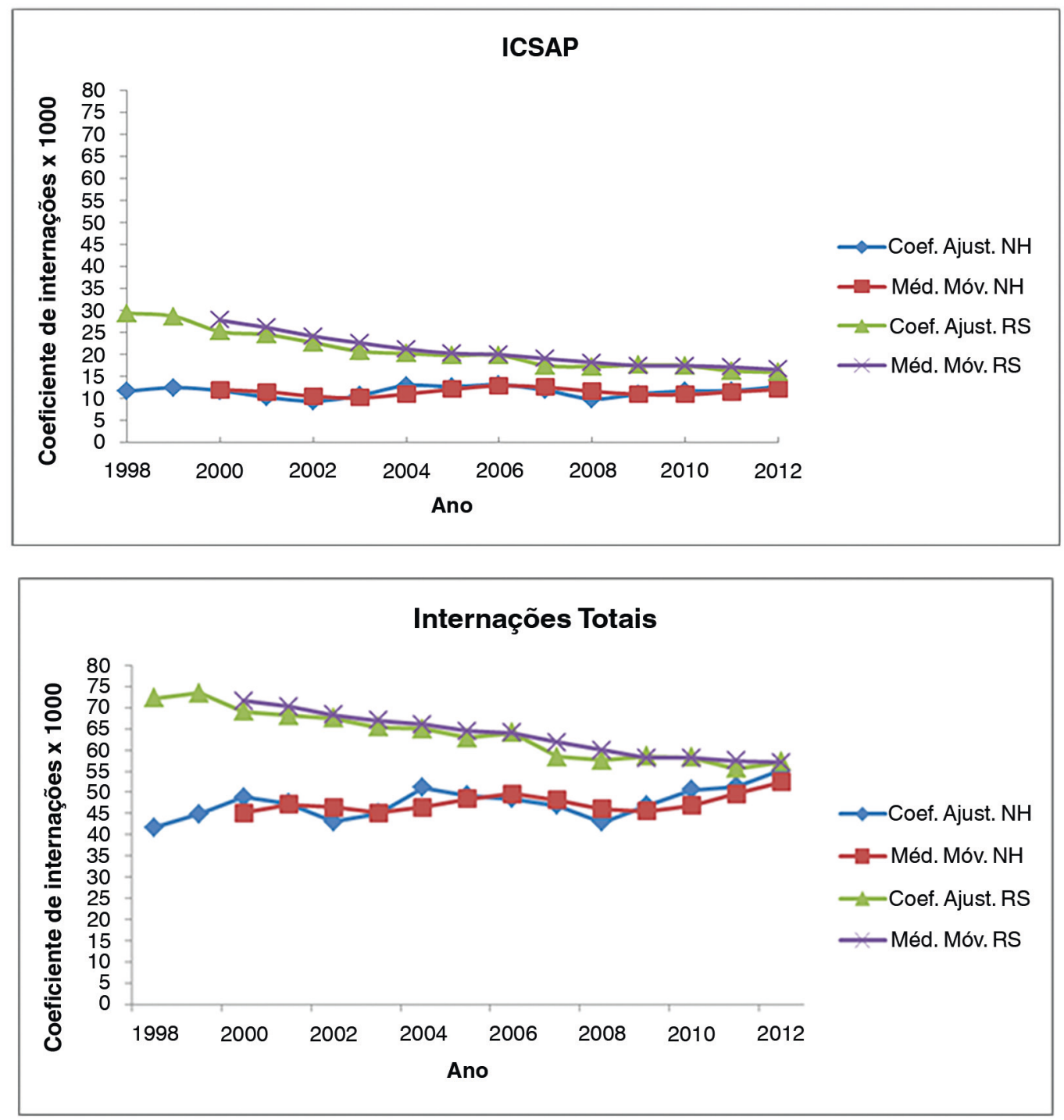

Gráfico 1. Coeficientes de ICSAP e coeficiente de internação por todas as causas padronizados com suas respectivas médias móveis. Rio Grande do Sul (sem Novo Hamburgo) e Novo Hamburgo, 1998-2012.

Em 1998, os custos das ICSAP atingiram $R \$ 3.216 .590,90$, passando para $R \$ 3.430 .535,00$ no ano de 2012, o que representou um aumento de 6,70\%. Entretanto, quando analisados os percentuais dos custos das ICSAP em relação aos investimentos financeiros totais, verificou-se que estes passaram de 10,55\% em 1998 para 2,62\% em 2012, representando uma redução de 75,17\% no período (Tabela 2).

Com esse estudo, observou-se que a análise da cobertura populacional pela ESF ficou prejudicada, pois o município aderiu à Estratégia de Saúde da Família somente no ano de 2011, partindo de 17,33\% no primeiro ano e atingindo $20,20 \%$ da população em 2012 (Tabela 2).

$\mathrm{Na}$ análise da Regressão Binomial Negativa, não foram encontradas diferenças entre as taxas de ICSAP no período estudado ( $p=0,87)$, assim como no investimento em APS $(p=0,29)$, porém percebeu-se um aumento significativo do investimento financeiro em saúde $(p<0,0001)$, do gasto per capita em saúde $(p<0,0001)$ e da cobertura pela ESF $(p=0,0009)$ no período estudado (Tabela 3$)$. 
Tabela 2. Investimento financeiro por ano, gasto per capita em saúde, investimento em atenção primária em saúde, percentual de investimento em atenção primária à saúde em relação ao investimento total em saúde, custo por ICSAP, percentual de custo por ICSAP em relação ao investimento total em saúde e cobertura populacional.

\begin{tabular}{|c|c|c|c|c|c|c|}
\hline Ano & $\begin{array}{c}\text { Investimento } \\
\text { Financeiro/ano (IF) }\end{array}$ & $\begin{array}{l}\text { Gasto per } \\
\text { Capita saúde }\end{array}$ & $\begin{array}{c}\text { Investimentos } \\
\text { APS/ano (IAPS) } \\
\text { em R\$ }\end{array}$ & $\begin{array}{c}\% \\
\text { IAPS/IF }\end{array}$ & $\begin{array}{l}\text { Custo } \\
\text { ICSAP } \\
\text { em R\$ }\end{array}$ & $\begin{array}{c}\% \text { Custo } \\
\text { ICS } \\
\text { P/IF }\end{array}$ \\
\hline 1998 & $\mathrm{R} \$ 30.500 .995,07$ & U\$ 32,66 & $0,00^{*}$ & $0,00^{*}$ & $3.216 .590,90$ & 10,55 \\
\hline 1999 & $\mathrm{R} \$ 20.706 .132,84$ & U\$ 17,03 & $0,00^{*}$ & $0,00^{*}$ & $3.142 .450,36$ & 15,18 \\
\hline 2000 & $\mathrm{R} \$ 33.224 .385,36$ & U\$ 30,09 & $0,00^{*}$ & $0,00^{*}$ & $2.757 .837,84$ & 8,30 \\
\hline 2001 & $\mathrm{R} \$ 41.789 .818,82$ & U\$ 32,05 & $0,00^{*}$ & $0,00^{*}$ & $2.245 .273,99$ & 5,37 \\
\hline 2003 & $\mathrm{R} \$ 36.935 .464,45$ & U\$ 30,34 & $25.847 .446,90$ & 69,98 & $1.907 .521,27$ & 5,16 \\
\hline 2004 & $\mathrm{R} \$ 61.422 .418,18$ & U\$ 51,71 & $26.030 .350,38$ & 42,38 & 2.311.345,39 & 3,76 \\
\hline 2005 & $\mathrm{R} \$ 65.600 .112,26$ & U\$ 72,26 & $27.026 .961,59$ & 41,20 & $2.179 .765,84$ & 3,32 \\
\hline 2006 & $\mathrm{R} \$ 74.904 .352,54$ & U\$ 91,64 & $27.274 .346,04$ & 36,41 & 2.193.622,00 & 2,92 \\
\hline 2010 & $\mathrm{R} \$ 101.671 .069,83$ & U\$ 208,68 & $80.383 .823,45$ & 79,06 & $2.767 .625,63$ & 2,72 \\
\hline 2011 & $\mathrm{R} \$ 126.127 .767,30$ & U\$ 315,07 & 107.913.630,37 & 85,59 & $2.774 .779,78$ & 2,20 \\
\hline 2012 & $\mathrm{R} \$ 130.929 .810,25$ & U\$ 275,02 & $119.990 .755,51$ & 91.64 & $3.430 .535,00$ & 2,62 \\
\hline
\end{tabular}

* Dados não disponíveis no SIOPS no referido período.

Tabela 3. Regressão Binomial Negativa da taxa padronizada de ICSAP, do investimento financeiro, do investimento em APS, do gasto per capita e da cobertura de ESF, em relação aos anos. Novo Hamburgo, 1998-2012.

\begin{tabular}{lccr}
\hline Variáveis & Razão de médias & IC $95 \%$ & p-valor \\
\hline ICSAP & 1,003 & 0,97 a 1,04 & 0,87 \\
IF/ano & 1,124 & 1,11 a 1,14 & $<0,0001$ \\
IF APS/ano & 1,345 & 0,60 a 2,97 & 0,29 \\
Gasto PC & 1,226 & 1,20 a 1,26 & $<0,0001$ \\
\hline
\end{tabular}

$\mathrm{Na}$ análise da correlação de Spearman, foi possível observar que todas as variáveis apresentaram associação positiva, porém, muito fraca, ou seja, mesmo com o aumento no investimento financeiro, no investimento em APS, no gasto per capita em saúde e na cobertura pela ESF, houve aumento discreto nos coeficientes de ICSAP, contudo, essas diferenças não foram estatisticamente significativas (Tabela 4).

Tabela 4. Correlação de Spearman das taxas padronizadas de ICSAP em relação ao investimento financeiro total em saúde, investimentos financeiros em atenção primária à saúde, gasto per capita em saúde e cobertura da ESF. Novo Hamburgo, 1998-2012.

\begin{tabular}{lccc} 
Variáveis & $\mathbf{R}$ & $\mathbf{r}^{2}$ & p-valor \\
\hline Investimento financeiro & 0,1036 & $1,07 \%$ & 0,71 \\
Investimento em APS & 0,2036 & $4,14 \%$ & 0,46 \\
Gasto per capita (dólar) & 0,0857 & $0,73 \%$ & 0,76 \\
Cobertura ESF & 0,1932 & $3,73 \%$ & 0,49 \\
\hline
\end{tabular}




\section{Discussão}

O presente estudo não mostrou diminuição nas tendências de ICSAP em Novo Hamburgo entre 1998 e 2012, como se demonstrou no restante do estado do Rio Grande do Sul, apesar de ter sido observado aumento nos investimentos financeiros em saúde e a adesão e expansão da cobertura da ESF no município.

Cabe ressaltar que Novo Hamburgo, por ser um polo industrial, por muito tempo teve grande parte da população com acesso a planos de saúde privados. Com a crise das empresas calçadistas e consequente restrição ao acesso a esses serviços, a população passou a procurar na rede básica municipal a solução para seus problemas, com isso, aumentando a demanda e, consequentemente, fazendo com que o município necessitasse um investimento maior em APS.

A análise do comportamento das tendências dos coeficientes seguiu uma estratégia baseada na comparação das ICSAP com as internações por todas as causas, na construção de médias móveis e na regressão binomial negativa. Apesar das discretas elevações percentuais encontradas nas comparações dos coeficientes, a regressão binomial não revelou diferenças significativas no período.

As diversas transformações ocorridas no Sistema Único de Saúde têm provocado diminuição das ICSAP em diferentes locais do Brasil. ${ }^{7-10,23}$

Uma das inovações que tem se mostrado relevante na diminuição das ICSAP tem sido a expansão da ESF. ${ }^{24}$ Estudos realizados em Minas Gerais mostraram uma relação do aumento da cobertura da ESF com a diminuição das ICSAP, bem como redução da probabilidade de internar nas áreas acompanhadas pela estratégia. Após a expansão da ESF, observou-se uma redução de 17,9\%, entre 2004 e 2006, em Belo Horizonte ${ }^{7}$. No município de Montes Claros, o controle regular fora da ESF duplicou a probabilidade de internação. ${ }^{25}$ Deve-se advertir que Belo Horizonte já contava com mais de $70 \%$ de cobertura de ESF em $2002^{7}$ e Montes Claros com aproximadamente 50\% no ano de 2007. ${ }^{25}$ Embora a ESF em Novo Hamburgo em dois anos tenha atingido $20,2 \%$, ainda deve ser considerada como incipiente quando comparada com critérios de classificação de cobertura populacional, não produzindo o impacto esperado na diminuição das ICSAP. ${ }^{26-28}$

Outro aspecto que deve ser destacado foi que a presente análise não permitiu verificar a origem dos pacientes que internaram por condições sensíveis, distinguindo acesso ou não à ESF.

Em relação aos investimentos financeiros, foi observado um incremento no período. Os dados referentes aos gastos per capita mostraram um aumento de mais de $740 \%$ no período de 15 anos, passando de $\bigcup \$ 32,66$ para $\bigcup \$ 275,02$, de acordo com cotação da moeda americana na metade do período de cada ano correspondente. Este valor foi inferior ao gasto per capita brasileiro, de U\$474,00, que tem sido denunciado como insuficiente por diversos autores. ${ }^{29}$ Por sua vez, ao se comparar com países latinoamericanos, estes valores se encontraram ainda mais abaixo, podendo-se citar como exemplos o gasto per capita de $\cup \$ 851,00$ na Argentina, U\$ 825,00 na Costa Rica e U\$ 740,00 no Uruguai, segundo dados de 2013. ${ }^{30}$ Isto pode ser explicado pelo fato de que os gastos públicos em saúde no Brasil estavam abaixo do praticado em outros países de média-baixa renda e alta renda. ${ }^{31}$ 
Ao se analisar o comportamento das despesas com APS em Novo Hamburgo, observou-se um aumento no investimento com o passar dos anos, destacando o ano de 2012, no qual $91 \%$ do total investido foram destinados à APS, provavelmente pela expansão da ESF, um montante relevante ao se comparar com os recursos federais, visto que o Brasil destinou $21 \%$ das despesas totais em saúde para a APS no período. ${ }^{30}$ Porém, ficou o questionamento se essas vultosas despesas em APS no referido ano foram efetivamente reais, visto que teriam sido aplicados somente $9 \%$ no nível hospitalar; talvez isso tenha ocorrido pela necessidade de praticamente se criar uma rede de atenção básica no município, que até então era incipiente.

Nesse sentido, um estudo que demonstrou os gastos com ICSAP na cidade de São José do Rio Preto, SP, município com cerca de 400 mil habitantes, revelou um valor de $R \$ 30.370 .691,08$, nos anos de 2008 a 2010. ${ }^{15}$ Nesse mesmo período os gastos de ICSAP em Novo Hamburgo foram de R $\$ 7.846 .941,61$. Embora não tivessem mesma população, ambos os municípios se enquadravam na mesma faixa do Programa Expansão e Consolidação Saúde da Família (PROESF), ou seja, entre 100.000 e 500.000 habitantes. Com esses dados, considerou-se que houve um menor gasto com ICSAP em Novo Hamburgo, do que em São José do Rio Preto no referido período.

Estudos ecológicos verificando tendências de séries históricas apresentam inúmeras limitações e, além dos problemas oriundos da utilização de dados secundários (subnotificação e erros de classificação), ${ }^{32}$ sofrem com variação aleatória dos coeficientes ${ }^{19}$ e pela análise agregada não controlam fatores de confusão. Entretanto, recente estudo realizado no Rio Grande do Sul mostrou a adequação do SIH/SUS, revelando a apropriação do uso das ICSAP. ${ }^{33}$ Em relação ao controle de fatores de confusão, na análise do presente estudo construíram-se médias móveis para "alisamento da tendência" e padronizaram-se os coeficientes ajustando o efeito de idade como tentativas de minimizar as limitações.

A realização de estudos tendo como fonte de dados o SIH/SUS certamente pode contribuir para seu aprimoramento, desde que utilizados para a avaliação, gestão e delineamento de políticas de saúde. Ao pensar que a regionalização é um princípio ordenador dos sistemas de saúde, pode-se apontar a necessidade de incorporação no sistema de informações de alguma variável mais proximal do local de residência dos indivíduos que possa identificar seus serviços de saúde de origem.

Cabe destacar que estudos que avaliam os coeficientes de ICSAP e suas tendências são importantes fontes de dados para o gerenciamento em saúde e para formulação de políticas e estratégias que visem à redução destes coeficientes, de modo a aperfeiçoar o sistema de saúde e os investimentos financeiros aplicados. Ressalta-se, ainda, que a criação da lista brasileira de ICSAP permite a comparabilidade entre estudos nacionais e contribui para a sua consolidação como indicador no país.

Contudo, embora no presente estudo não tenha sido encontrada associação entre a diminuição nos coeficientes de ICSAP com o aumento da cobertura pela ESF, incremento do investimento financeiro em saúde, investimento financeiro em APS e o gasto per capita, há evidências que estes estejam correlacionados. ${ }^{7,34}$ Recomendam-se outros estudos que permitam identificar fatores individuais relacionados às internações hospitalares como identificação do serviço de origem e condições socioeconômicas, além do acompanhamento contínuo das ICSAP, que se revelam como indicadores disponíveis e rápidos para reorganização e aprimoramento do sistema de saúde. 


\section{Referências}

1. Billings J, Zeitel L, Lukomnik J, Carey TS, Blank AE, Newman L. Impact of socioeconomic status on hospital use in New York City. Health Aff (Millwood). 1993;12(1):162-73. DOI: http://dx.doi.org/10.1377/hlthaff.12.1.162

2. Gill JM. Can hospitalizations be avoided by having a regular source of care? Fam Med. 1997;29(3):166-71.

3. Casanova C, Starfield B. Hospitalizations of children and access to primary care: a cross-national comparison. Int $\mathrm{J}$ Health Serv. 1995;25(2):283-94. DOI: http://dx.doi.org/10.2190/PCF7-ALX9-6CN3-7X9G

4. Roos NP, Mustard CA. Variation in health and health care use by socioeconomic status in Winnipeg, Canada: does the system work well? Yes and no. Milbank Q. 1997;75(1):89-111. DOI: http://dx.doi.org/10.1111/1468-0009.00045

5. Shi L, Samuels ME, Pease M, Bailey WP, Corley EH. Patient characteristics associated with hospitalizations for ambulatory care sensitive conditions in South Carolina. South Med J. 1999;92(10):989-98. DOI: http://dx.doi.org/10.1097/00007611199910000-00009

6. Alfradique ME, Bonolo PF, Dourado I, Lima-Costa MF, Macinko J, Mendonça CS, et al. Internações por condições sensíveis à atenção primária: a construção da lista brasileira como ferramenta para medir o desempenho do sistema de saúde (Projeto ICSAP - Brasil). Cad Saúde Pública. 2009;25(6):1337-49. DOI: http://dx.doi.org/10.1590/S0102-311X2009000600016

7. Mendonça CS, Harzheim E, Duncan BB, Nunes LN, Leyh W. Trends in hospitalizations for primary care sensitive conditions following the implementation of Family Health Teams in Belo Horizonte, Brazil. Health Policy Plan. 2012;27(4):348-55. DOI: http://dx.doi.org/10.1093/heapol/czr043

8. Batista SRR, Jardim PCBV, Sousa ALL, Salgado CM. Hospitalizações por condições cardiovasculares sensíveis à atenção primária em municípios goianos. Rev Saúde Pública. 2012;46(1):34-42. DOI: http://dx.doi.org/10.1590/S003489102012005000001

9. Souza LL, Costa JSD. Internações por condições sensíveis à atenção primária nas coordenadorias de saúde no RS. Rev Saúde Pública. 2011;45(4):765-72. DOI: http://dx.doi.org/10.1590/S0034-89102011000400017

10. Barreto JOM, Nery IS, Costa MSC. Estratégia de Saúde da Família e internações hospitalares em menores de 5 anos no Piauí, Brasil. Cad Saúde Pública. 2012;28(3):515-26. DOI: http://dx.doi.org/10.1590/S0102-311X2012000300012

11. Campbell SM, Roland MO, Buetow SA. Defining quality of care. Soc Sci Med. 2000;51(11):1611-25. DOI: http://dx.doi. org/10.1016/S0277-9536(00)00057-5

12. Mendes A. A longa batalha pelo financiamento do SUS. Saúde Soc. 2013;22(4):987-93. DOI: http://dx.doi.org/10.1590/ S0104-12902013000400002

13. Mendes EV. O SUS que temos e o SUS que queremos: uma agenda. Rev Min Saúde Pública. 2004;3(4):4-26.

14. Mendes EV. As redes de atenção à saúde. $2^{2}$ ed. Brasília: Organização Pan-Americana da Saúde; 2011.

15. Ferreira JBB, Borges MJG, Santos LL, Forster AC. Internações por condições sensíveis à atenção primária à saúde em uma região de saúde paulista, 2008 a 2010. Epidemiol Serv Saúde. 2014;23(1):45-56. DOI: http://dx.doi.org/10.5123/ S1679-49742014000100005

16. Rehem TCMSB, Egry EY. Internações por condições sensíveis à atenção primária no estado de São Paulo. Ciênc Saúde Coletiva. 2011;16(12):4755-66. DOI: http://dx.doi.org/10.1590/S1413-81232011001300024

17. Lilienfeld AM, Lilienfeld DE. Foundations of epidemiology. 2nd ed. New York: Oxford University Press; 1980.

18. Latorre MRDO, Cardoso MRA. Análise de séries temporais em epidemiologia: uma introdução sobre os aspectos metodológicos. Rev Bras Epidemiol. 2001;4(3):145-52. DOI: http://dx.doi.org/10.1590/S1415-790X2001000300002

19. Oliveira G, Pacheco M. Mercado Financeiro: Objetivo e Profissional. Curitiba: Fundamento; 2006.

20. Amado AM, Mollo MLR. Noções de macroeconomia: razões teóricas para as divergências entre economistas. São Paulo: Manole; 2003.

21. Rosenberg D. Trend analysis and interpretation. Key concepts and methods for maternal and child health professionals. Rockville: Division of Science, Education and Analysis Maternal and Child Health Bureau; 1997.

22. Chen PY, Popovich PM. Correlation: Parametric and Nonparametric Measures. Thousand Oaks: Sage Publication; 2002. 
23. Macinko J, de Oliveira VB, Turci MA, Guanais FC, Bonolo PF, Lima-Costa MF. The influence of primary care and hospital supply on ambulatory care-sensitive hospitalizations among adults in Brazil, 1999-2007. Am J Public Health. 2011;101(10):1963-70. DOI: http://dx.doi.org/10.2105/AJPH.2010.198887

24. Campos AZ, Theme-Filha MM. Internações por condições sensíveis à atenção primária em Campo Grande, Mato Grosso do Sul, Brasil, 2000 a 2009. Cad Saúde Pública. 2012;28(5):845-55. DOI: http://dx.doi.org/10.1590/S0102311X2012000500004

25. Fernandes VBL, Caldeira AP, Faria AA, Rodrigues Neto JF. Internações sensíveis na atenção primária como indicador de avaliação da Estratégia Saúde da Família. Rev Saúde Pública. 2009;43(6):928-36. DOI: http://dx.doi.org/10.1590/S003489102009005000080

26. Figueiró AC, Hartz ZM, Thuler LC, Dias AL. Meta-evaluation of baseline studies of the Brazilian Family Health Strategy Expansion Project: a participatory and formative approach. Cad Saude Pública. 2010;26(12):2270-8. DOI: http://dx.doi. org/10.1590/S0102-311X2010001200006

27. Carvalho SC, Mota E, Dourado I, Aquino R, Teles C, Medina MG. Hospitalizations of children due to primary health care sensitive conditions in Pernambuco State, Northeast Brazil. Cad Saude Pública. 2015;31(4):744-54. DOI: http://dx.doi. org/10.1590/0102-311X00069014

28. Aquino R, de Oliveira NF, Barreto ML. Impact of the family health program on infant mortality in Brazilian municipalities. Am J Public Health. 2009;99(1):87-93. DOI: http://dx.doi.org/10.2105/AJPH.2007.127480

29. Santos NR. SUS, política pública de Estado: seu desenvolvimento instituído e instituinte e a busca de saídas. Ciênc Saúde Coletiva. 2013;18(1):273-80. DOI: http://dx.doi.org/10.1590/S1413-81232013000100028

30. Mendes EV. 25 anos do Sistema Único de Saúde: resultados e desafios. Estud Avanç. 2013;27(78):27-34. DOI: http:// dx.doi.org/10.1590/S0103-40142013000200003

31. Macinko J, Harris MJ. Brazil's family health strategy--delivering community-based primary care in a universal health system. N Engl J Med. 2015;372(23):2177-81. DOI: http://dx.doi.org/10.1056/NEJMp1501140

32. Bittencourt SA, Camacho LAB, Leal MC. O Sistema de Informação Hospitalar e sua aplicação na saúde coletiva. Cad Saúde Pública. 2006;22(1):19-30. DOI: http://dx.doi.org/10.1590/S0102-311X2006000100003

33. Abaid RA. Análise da confiabilidade dos diagnósticos das autorizações de internação hospitalar: um estudo em Santa Cruz do Sul [Dissertação de mestrado]. Santa Cruz do Sul: Universidade de Santa Cruz do Sul (UNISC); 2012.

34. Brasil VP, Costa JSD. Hospitalizações por condições sensíveis à atenção primária em Florianópolis, Santa Catarina estudo ecológico de 2001 a 2011. Epidemiol Serv Saúde. 2016;25(1):75-84.

a Universidade do Vale do Rio dos Sinos (UNISINOS), PPG em Saúde Coletiva. São Leopoldo, RS, Brasil. doc.arruda@ hotmail.com (Autor correspondente); episoares@terra.com.br 